\title{
Tarlatt
}

Implementierung von Strategien im Unternehmen 
GABLER EDITION WISSENSCHAFT 


\section{Alexander Tarlatt}

\section{Implementierung \\ von Strategien \\ im Unternehmen}

Mit einem Geleitwort

von Prof. Dr. Franz Eisenführ

Deutscher Universitäts-Verlag 
Die Deutsche Bibliothek - CIP-Einheitsaufnahme

Tarlatt, Alexander:

Implementierung von Strategien im Unternehmen / Alexander Tarlatt. Mit einem Geleitw. von Franz Eisenführ. - 1. Aufl. - Wiesbaden : Dt. Univ.-Verl. ; Wiesbaden : Gabler, 2001

(Gabler Edition Wissenschaff)

Zugl.: Köln, Univ., Diss., 2000

1. Auflage August 2001

Alle Rechte vorbehalten

(C) Betriebswirtschaftlicher Verlag Dr. Th. Gabler GmbH, Wiesbaden, und

Deutscher Universitäts-Verlag GmbH, Wiesbaden, 2001

Lektorat: Brigitte Siegel / Stefanie Loyal

Der Gabler Verlag und der Deutsche Universitäts-Verlag sind Unternehmen der Fachverlagsgruppe BertelsmannSpringer.

www.gabler.de

unw.duv.de

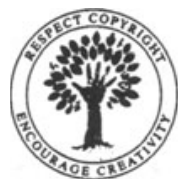

Das Werk einschließlich aller seiner Teile ist urheberrechtlich geschützt. Jede Verwertung außerhalb der engen Grenzen des Urheberrechtsgesetzes ist ohne Zustimmung des Verlages unzulässig und strafbar. Das gilt insbesondere für Vervielfältigungen, Übersetzungen, Mikroverfilmungen und die Einspeicherung und Verarbeitung in elektronischen Systemen.

Die Wiedergabe von Gebrauchsnamen, Handelsnamen, Warenbezeichnungen usw. in diesem Werk berechtigt auch ohne besondere Kennzeichnung nicht zu der Annahme, dass solche Namen im Sinne der Warenzeichen- und Markenschutz-Gesetzgebung als frei zu betrachten wären und daher von jedermann benutzt werden dürften.

Gedruckt auf säurefreiem und chlorfrei gebleichtem Papier. 


\section{Geleitwort}

Voraussetzung für den Erfolg einer Unternehmensstrategie ist ihre effektive Implementierung. Tarlatt geht von der durch empirische Beobachtungen gestützten Annahme aus, dass ein großer Teil neu entwickelter Strategien an unzulänglicher Umsetzung scheitert oder dass die Umsetzung auf wenig effiziente Art erfolgt. Ziel der Arbeit ist die Entwicklung einer systematischen Vorgehensweise, die die Effektivität und Effizienz der Strategie-Implementierung verbessern soll.

Die Kernidee ist, dass Implementierungsempfehlungen nicht unabhängig von inhaltlichen Merkmalen der zu implementierenden Strategien gegeben werden können. So definiert der Verfasser Strategieklassen und untersucht, wie diese sich in Bezug auf Implementierungsziele, Implementierungsprinzipien, organisatorische Gestaltung der Implementierung und Implementierungsmaßnahmen voneinander unterscheiden.

Als mögliche Implementierungsziele werden Implementierungsgeschwindigkeit, Akzeptanz der Strategie bei Betroffenen, Konsens zwischen Entscheidern und Partizipation der Betroffenen identifiziert. Implementierungsprinzipien unterscheidet der Verfasser entlang einer Richtungs- und einer Intensitätsdimension. Richtungsmäßig wird zwischen Top-down, Bottom-up und From-the-Middle unterschieden. Nach der Intensität der Implementierung unterscheidet der Verfasser zwischen inkrementeller, radikaler, stufenweiser, progressiver und degressiver Implementierung. Als Implementierungsmaßnahmen werden Kommunikations-, Organisations-, Motivations- und Unterstützungsmaßnahmen unterschieden.

Die Arbeit behandelt eine Thematik, die einerseits in hohem Maße praktisch bedeutungsvoll ist und andererseits wissenschaftlich in dieser Breite noch nicht behandelt wurde. Sie erhält besonderen Wert durch eine explorative Befragung, die der Verfasser unter TopFührungskräften von Großunternehmen durchgeführt hat. Die Ergebnisse sind, sorgfältig aufbereitet und übersichtlich dargestellt, so in den Text integriert, dass Hypothesen und empirische Ergebnisse unmittelbar nebeneinander stehen.

Der Stand der Implementationsforschung ist in Tarlatts Arbeit umfassend und gründlich aufgearbeitet. Für das strategische Management enthält die Schrift eine Fülle fundierter Anregungen. 


\section{Vorwort}

Die vorliegende Arbeit, die im Sommersemester 2001 von der Wirtschafts- und Sozialwissenschaftlichen Fakultät der Universität zu Köln als Dissertation angenommen worden ist, behandelt die Problematik der Implementierung von Strategien im Unternehmen. Da eine hohe Anzahl von Umsetzungsversuchen scheitert, erschien die Behandlung dieser Thematik sowohl vor wissenschaftlichem als auch praktischem Hintergrund interessant. In der Literatur zum strategischen Management werden Implementierungsaspekte meist nur am Rande der Strategieformulierung gestreift, um die Notwendigkeit einer Umsetzung der entwickelten Strategie hervorzuheben. Arbeiten, in deren Mittelpunkt die Implementierung von Strategien steht, sind in einer überschaubaren Anzahl vorhanden. Inhalt dieser Arbeiten ist oft die Beschreibung und Analyse der Implementierung einer bestimmten Strategie oder eines bestimmten Strategietyps.

Die vorliegende Arbeit ist das Ergebnis einer gründlichen Auseinandersetzung mit dieser Problemstellung, die über eine Einzelfallbetrachtung nur eines bestimmten Strategietyps hinausgeht. Die Implementierung von Strategien erfolgt hier mittels eines prozessualen Vorgehens, welches theoretisch abgeleitet wurde und sich praktisch als zweckmäßig erweist. In der Arbeit werden Merkmale von Strategien als Entscheidungskriterien verwendet. Die Kombination der Merkmale erlaubt die Abbildung vieler in der Realität vorhandener Strategien, was die Anwendbarkeit des Phasenkonzeptes erhöht. Zur Stärkung der aufgestellten Thesen ist im Rahmen dieser Arbeit eine empirische Untersuchung durchgeführt worden.

Ich hoffe, dass das Feld der Implementierungsforschung im Rahmen des strategischen Managements bald stärker bearbeitet wird. Der Wert einer Strategie für ein Unternehmen entwickelt sich schließlich erst durch deren erfolgreiche und effiziente Umsetzung.

Ich möchte an dieser Stelle herzlich all denen danken, die mich bei der Erstellung dieser Arbeit unterstützt und motiviert haben. Vor allem gilt mein Dank den Teilnehmern der empirischen Untersuchung, die dazu bereit waren, ihr Wissen und ihre Erfahrung in Interviews oder durch das Ausfüllen eines Fragebogens für diese Arbeit zugänglich zu machen.

Darüber hinaus möchte ich mich bei meinem Doktorvater Prof. Dr. Franz Eisenführ bedanken, der mich bei der Erstellung der Dissertation stets unterstützt hat. Die jederzeit möglichen konstruktiven Gespräche, die ausgezeichnete Betreuung und der gewährte Freiraum waren eine wichtige Voraussetzung für das Entstehen dieser Arbeit.

Prof. Dr. Erich Frese gilt mein Dank für die Übernahme des Korreferats.

$\mathrm{Zu}$ Dank verpflichtet bin ich auch meinen Kollegen vom Seminar für Allgemeine Betriebswirtschaftslehre, Dipl.-Kfm. Kristian Foit, Dipl.-Kfm. Marc Kastner, Dipl.-Kff. 
Natalia Nikolova, Dr. rer. pol. Antonio Vera sowie allen Hilfskräften für ihre Unterstützung und die konstruktiven Gespräche.

Insbesondere danke ich meinen Eltern, die mich während meiner gesamten Ausbildungszeit in vielerlei Hinsicht unterstützt und somit erst das Entstehen dieser Arbeit ermöglicht haben. Sie motivierten mich kontinuierlich, meine gesetzten Ziele zu erreichen. Ihnen sei diese Arbeit gewidmet.

Schließlich möchte ich mich bei meiner Freundin Michaela bedanken, die mich in der vergangenen Zeit stetig ermutigte und mir in schwierigen Phasen immer liebevollen Rückhalt gab. 


\section{Inhaltsverzeichnis}

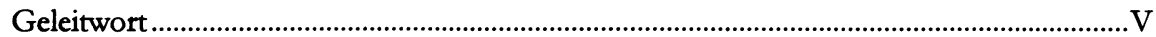

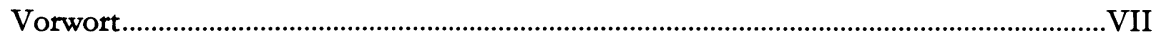

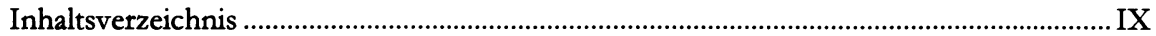

Abbildungsverzeichnis ...........................................................................................................XIII

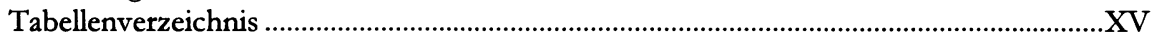

Abkürzungsverzeichnis ................................................................................................. XVII

1 Überblick und Einführung in die Themenstellung ................................................

1.1 Problematik der Strategieimplementierung ………....................................................

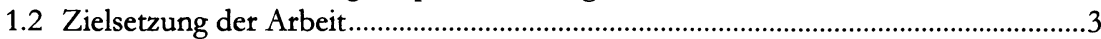

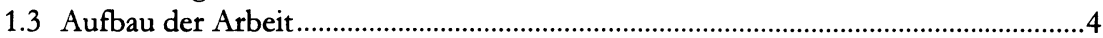

1.4 Explorative Erhebung ……………………………................................................

2 Theoretische Grundlagen .......................................................................................

2.1 Definition und Systematisierung von Strategien.........................................................

2.1.1 Definition der Strategie ................................................................................ 10

2.1.2 Systematisierung des Strategiespektrums...................................................... 14

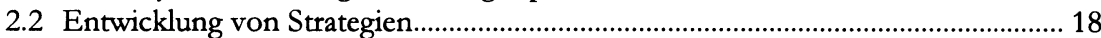

2.2.1 Zielplanung als Voraussetzung für die Strategieentwicklung......................... 20

2.2.1.1 Auswirkung der Zielplanung auf die Strategieimplementierung.... 24

2.2.1.2 Ziele der Unternehmung ...................................................................... 25

2.2.1.2.1 Basisziele …………………………………………….... 27

2.2.1.2.2 Geschäftsbereichsziele ...................................................... 28

2.2.1.2.3 Funktionsbereichsziele................................................... 29

2.2.1.2.4 Instrumentalziele............................................................ 29

2.2.1.3 Ziele der Strategie .............................................................................. 29

2.2.1.4 Analyse der Zielkonflikte ................................................................... 30

2.2.2 Analyse der Potenziale vor dem Hintergrund der Implementierung........... 30

2.2.2.1 Finanzpotenzial................................................................................... 32

2.2.2.2 Organisationspotenzial ..................................................................... 33

2.2.2.3 Personalpotenzial ................................................................................ 35

2.2.3 Bewertung der Strategiealternativen ................................................................ 36

2.2.3.1 Allgemeine Funktion der Strategiebewertung .................................. 36

2.2.3.2 Grundmodell der Strategiebewertung von Rappaport ........................ 37

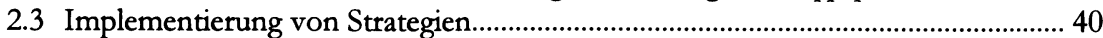

2.3.1 Definition der Strategieimplementierung......................................................... 41

2.3.2 Aufgaben der Strategieimplementierung......................................................... 43

2.3.3 Abgrenzung zum Change-Management......................................................... 45

2.3.4 Probleme der Strategieimplementierung........................................................ 46

2.3.5 Ablauf der Implementierung ...................................................................... 53

2.3.5.1 Implementierung aus der Unternehmensebene ............................... 55

2.3.5.2 Implementierung aus der Geschäftsbereichsebene ......................... 56

2.3.5.3 Implementierung aus der Funktionsbereichsebene .......................... 57 
2.4 Implementierungsrelevante psychologische Aspekte............................................... 59

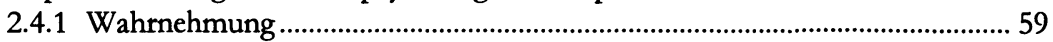

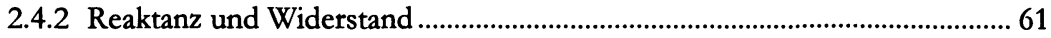

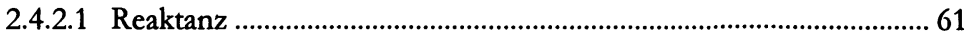

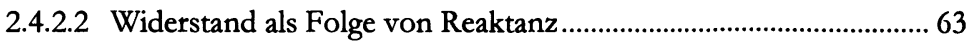

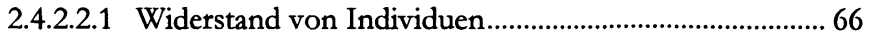

2.4.2.2.2 Widerstand von Gruppen.............................................. 70

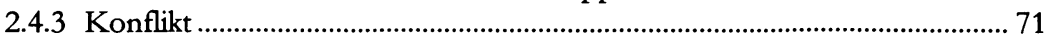

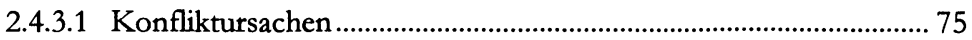

2.4.3.1.1 Konflikte innerhalb von Einheiten .................................... 76

2.4.3.1.2 Konflikte zwischen Einheiten........................................... 78

2.4.3.2 Konfliktlösungsansätze................................................................. 81

2.4.4 Konsequenzen der psychologischen Aspekte für die Implementierung ..... 86

2.5 Einflusskomponenten der Gestaltung des Implementierungsprozesses.................. 87

2.5.1 Entscheidungstheoretischer Einfluss ............................................................... 88

2.5.2 Phasen-Theorem der Entscheidung nach Witte _.......................................... 89

2.5.3 Phasenanalyse nach Kosiol........................................................................... 91

2.5.4 Lewins Triade .............................................................................................. 92

2.6 Konsequenzen für den Prozess der Strategieimplementierung............................... 92

3 Systematisierung von Strategiemerkmalen............................................................... 97

3.1 Ziele im Wandel - MIT-Studie ................................................................................... 97

3.2 Empirisch getestete Strategietypen der PIMS-Forschung......................................... 99

3.3 Aggregation der Aussagen der MIT-Studie und der PIMS-Forschung ................. 102

3.4 Entscheidungsrelevante Strategiemerkmale ……………………………………...... 105

3.4.1 Differenzierungsorientierung .................................................................... 107

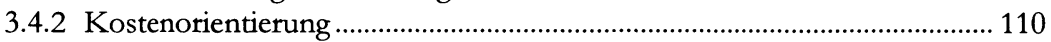

3.4.3 Qualitätsorientierung ................................................................................... 111

3.4.4 Zeitorientierung........................................................................................ 113

3.4.5 Expansionsorientierung............................................................................... 114

3.4.6 Desinvestitionsorientierung......................................................................... 116

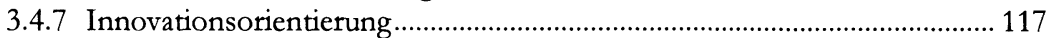

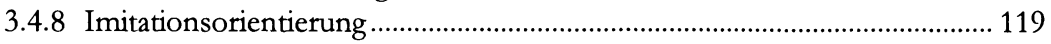

4 Analyse der Veränderungen und des Implementierungsumfeldes...................... 121

4.1 Analyse der Implementierungskomplexität ........................................................... 122

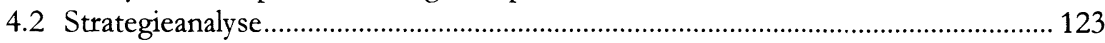

4.2.1 Strategieidentifikation ………………………………………………….... 123

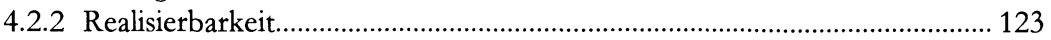

4.3 Analyse der Unternehmensgrößenveränderung ……………………………........ 125

4.4 Analyse der Marktfeldveränderung...................................................................... 125

4.5 Analyse der Organisationsstrukturveränderung...................................................... 126

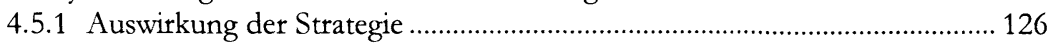

4.5.2 Auswirkung der Unternehmensgröße ........................................................ 128

4.5.3 Auswirkung der Technologie ................................................................... 128

4.5.4 Auswirkung der Umwelt ............................................................................... 130 
4.6 Beteiligtenanalyse

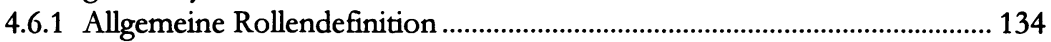

4.6.2 Rollen im Implementierungsprozess ............................................................ 136

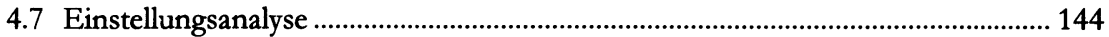

4.8 Entwicklung operationaler Zwischenziele ................................................................ 146

5 Realisation der Implementierung....................................................................... 151

5.1 Ziele bei der Implementierung von Strategien...................................................... 152

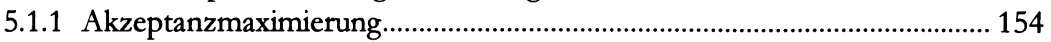

5.1.2 Konsens zwischen beteiligten Personen oder Bereichen ............................. 155

5.1.3 Starke Partizipation der Betroffenen an der Implementierung.................... 158

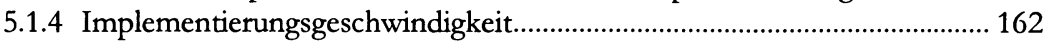

5.1.5 Zusammenfassende Gegenüberstellung ...................................................... 165

5.2 Implementierungsprinzipien als Grundsatzentscheidung ……………………..... 167

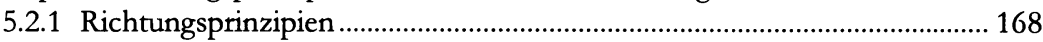

5.2.1.1 Top-Down-Implementierung .......................................................... 169

5.2.1.2 Bottom-Up-Implementierung ………………............................... 173

5.2.1.3 From-the-Middle-Implementierung …………………………..... 177

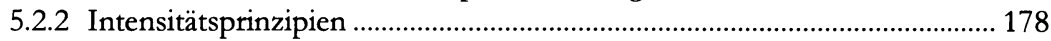

5.2.2.1 Inkrementelle Implementierung ................................................... 179

5.2.2.2 Radikale Implementierung ……………………………………...... 182

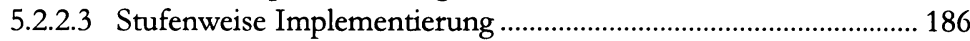

5.2.2.4 Progressive Implementierung ......................................................... 187

5.2.2.5 Degressive Implementierung …………………………………....... 191

5.2.3 Zusammenfassende Gegenüberstellung …………………………………... 193

5.3 Organisatorische Ausgestaltung der Implementierung............................................ 201

5.3.1 Integration des Implementierungsprojekts...................................................... 202

5.3.1.1 Bestehende Organisationsstruktur ................................................... 204

5.3.1.2 Stabs-Projektorganisation................................................................. 205

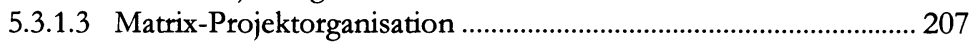

5.3.1.4 Reine Projektorganisation ................................................................ 210

5.3.2 Interne Organisation des Implementierungsprojekts................................... 212

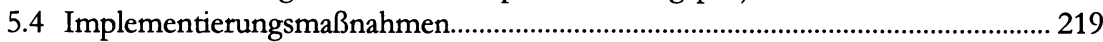

5.4.1 Kommunikationsmaßnahmen................................................................... 221

5.4.1.1 Maßnahmen der Abwärtskommunikation ....................................... 224

5.4.1.2 Maßnahmen der Dialogkommunikation ....................................... 229

5.4.1.3 Maßnahmen der Aufwärtskommunikation...................................... 233

5.4.1.4 Kommunikationsverzicht.............................................................. 236

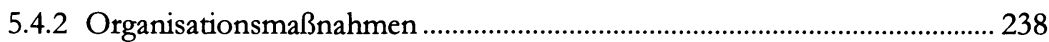

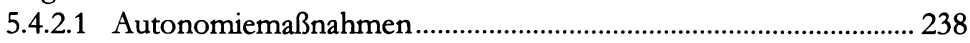

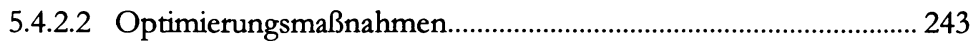

5.4.2.3 Verhaltensänderungsmaßnahmen .................................................. 248

5.4.2.4 Personalveränderungsmaßnahmen ................................................. 250

5.4.2.5 Formalisierungsmaßnahmen......................................................... 253 
5.4.3 Motivationsmaßnahmen......................................................................... 256

5.4.3.1 Partizipationsmaßnahmen........................................................... 260

5.4.3.2 Anreizmaßnahmen ............................................................................ 263

5.4.3.3 Bestrafungsmaßnahmen ................................................................ 271

5.4.3.4 Emotionale Konkretisierungsmaßnahmen .................................... 273

5.4.4 Unterstützungsmaßnahmen............................................................................ 278

5.4.4.1 Individualunterstützungsmaßnahmen ........................................... 280

5.4.4.2 Gruppenunterstützungsmaßnahmen ............................................. 285

5.4.4.3 Beratungsmaßnahmen ..................................................................... 288

5.5 Rechtliche Restriktionen und Vorschriften .......................................................... 290

5.6 Spezifisches Maßnahmenprogramm .................................................................. 299

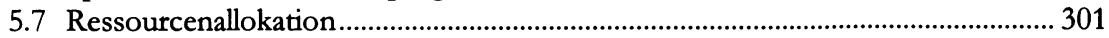

5.8 Kontrollvorbereitung .............................................................................................. 302

5.8.1 Verwendung von Meilensteinen...................................................................... 303

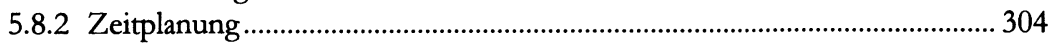

5.8.2.1 Grundlagen der Zeitplanung.......................................................... 304

5.8.2.2 Zeitaspekte der Maßnahmendimensionen........................................ 307

5.8.2.3 Methoden der Zeitplanung ……………………............................. 309

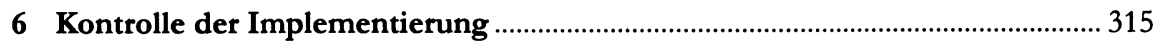

6.1 Kontrollorgane ......................................................................................................... 317

6.2 Kontrollarten ......................................................................................................... 318

6.3 Prozess der Implementierungskontrolle ………………........................................ 320

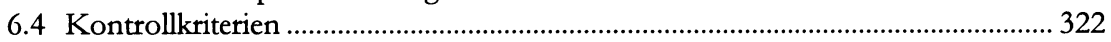

7 Analyse erforderlicher Anpassungen ...................................................................... 327

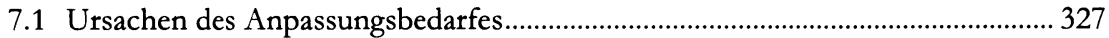

7.1.1 Unternehmensinduzierte Ursachen ................................................................. 327

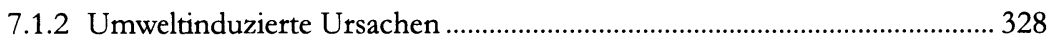

7.2 Alternativen der Anpassung ……………………….............................................329

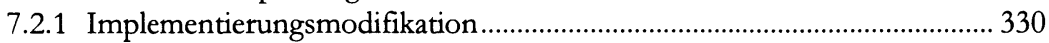

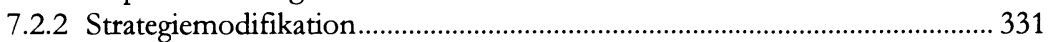

7.2.3 Strategieelimination............................................................................... 331

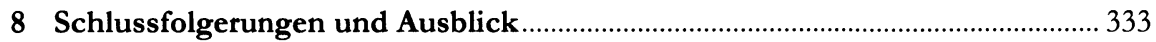

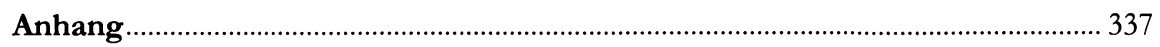

Anhang I: Fragebogen der explorativen Erhebung ..................................................... 339

Anhang II: Mittelwerte und Standardabweichungen der Ergebnisse .......................... 353

Anhang III: Prozentuale Häufigkeitsverteilungen der Ergebnisse .................................. 355

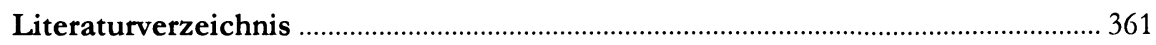




\section{Abbildungsverzeichnis}

Abbildung 1-1: Systematik der Erhebungsblöcke................................................................

Abbildung 2-1: Detailliertheit und Entscheidungsfeld von Strategien ............................ 17

Abbildung 2-2: Unternehmen und Koalitionsgruppen................................................... 22

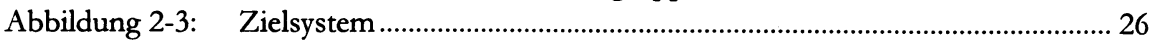

Abbildung 2-4: Implementierungspotenzial................................................................. 32

Abbildung 2-5: Handlungsfeld der Strategieimplementierung......................................... 46

Abbildung 2-6: Implementierungsablauf ....................................................................... 54

Abbildung 2-7: Phasen-Theorem der Entscheidung........................................................ 90

Abbildung 2-8: Aufgabengliederung in der Implementierung ...................................... 93

Abbildung 2-9: Der Implementierungsprozess............................................................. 95

Abbildung 3-1: Strategiemerkmale im Implementierungsprozess..................................... 97

Abbildung 3-2: Ableitung von Strategiemerkmalen ...................................................... 99

Abbildung 3-3: Erfahrung bei der Implementierung von Strategien............................. 105

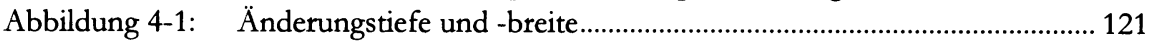

Abbildung 4-2: Rollen im Implementierungsprozess...................................................... 136

Abbildung 5-1: Erfolgskennlinien der Implementierung............................................... 151

Abbildung 5-2: Implementierungsziele ……………….............................................. 153

Abbildung 5-3: Beachtung der Akzeptanz als Zielsetzung........................................... 155

Abbildung 5-4: Beachtung des Konsens als Zielsetzung................................................ 157

Abbildung 5-5: Beachtung der Partizipation als Zielsetzung ........................................ 162

Abbildung 5-6: Beachtung der Implementierungsgeschwindigkeit als Zielsetzung..... 164

Abbildung 5-7: Zielgewichtung bei unterschiedlichen Strategiemerkmalen .................. 166

Abbildung 5-8: Allokation der Entscheidungskompetenz ………………………......... 169

Abbildung 5-9: Schematische Darstellung der Top-Down-Implementierung ……...... 170

Abbildung 5-10: Eignung der Top-Down-Implementierung............................................ 173

Abbildung 5-11: Schematische Darstellung der Bottom-Up-Implementierung.............. 174

Abbildung 5-12: Eignung der Bottom-Up-Implementierung .......................................... 176

Abbildung 5-13: Schematische Darstellung der From-the-Middle-Implementierung... 177

Abbildung 5-14: Eignung der From-the-Middle-Implementierung ................................. 178

Abbildung 5-15: Schematische Darstellung der inkrementellen Implementierung......... 180

Abbildung 5-16: Eignung der inkrementellen Implementierung....................................... 181

Abbildung 5-17: Schematische Darstellung der radikalen Implementierung................... 182

Abbildung 5-18: Eignung der radikalen Implementierung............................................... 185

Abbildung 5-19: Schematische Darstellung der stufenweisen Implementierung............ 186

Abbildung 5-20: Eignung der stufenweisen Implementierung ......................................... 187

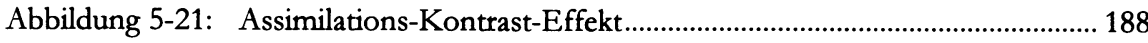

Abbildung 5-22: Schematische Darstellung der progressiven Implementierung ............ 189

Abbildung 5-23: Eignung der progressiven Implementierung........................................... 190

Abbildung 5-24: Schematische Darstellung der degressiven Implementierung ............. 191 
Abbildung 5-25: Eignung der degressiven Implementierung. 192

Abbildung 5-26: Implementierungsprinzipien bei kostenorientierten Strategien.

Abbildung 5-27: Implementierungsprinzipien bei differenzierungsorientierten Strategien... 195

Abbildung 5-28: Implementierungsprinzipien bei qualitätsorientierten Strategien........ 196

Abbildung 5-29: Implementierungsprinzipien bei zeitorientierten Strategien .................. 197

Abbildung 5-30: Implementierungsprinzipien bei expansionsorientierten

Strategien

Abbildung 5-31: Implementierungsprinzipien bei desinvestitionsorientierten Strategien...

Abbildung 5-32: Implementierungsprinzipien bei innovationsorientierten Strategien.. 200

Abbildung 5-33: Implementierungsprinzipien bei imitationsorientierten Strategien. 201

Abbildung 5-34: Eignung der bestehenden Organisationsstruktur................................. 205

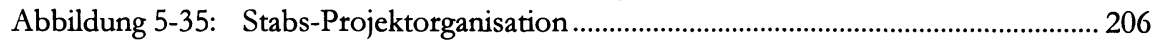

Abbildung 5-36: Eignung der Stabs-Projektorganisation ................................................. 207

Abbildung 5-37: Matrix-Projektorganisation................................................................ 208

Abbildung 5-38: Eignung der Matrix-Projektorganisation............................................... 209

Abbildung 5-39: Reine Projektorganisation.................................................................. 211

Abbildung 5-40: Eignung der reinen Projektorganisation................................................ 212

Abbildung 5-41: Kommunikationsprozess ....................................................................... 223

Abbildung 5-42: Eignung der Maßnahme Abwärtskommunikation................................. 225

Abbildung 5-43: Eignung der Maßnahme Dialogkommunikation.................................... 230

Abbildung 5-44: Eignung der Maßnahme Aufwärtskommunikation ............................... 233

Abbildung 5-45: Eignung der Maßnahme Kommunikationsverzicht............................. 237

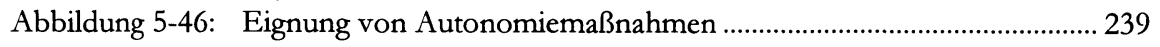

Abbildung 5-47: Eignung von Optimierungsmaßnahmen ................................................ 245

Abbildung 5-48: Eignung von Verhaltensänderungsmaßnahmen.................................... 249

Abbildung 5-49: Eignung von Personalveränderungsmaßnahmen................................. 251

Abbildung 5-50: Eignung von Formalisierungsmaßnahmen ......................................... 254

Abbildung 5-51: Eignung von Partizipationsmaßnahmen ................................................ 260

Abbildung 5-52: Eignung von Anreizmaßnahmen........................................................ 264

Abbildung 5-53: Eignung von Bestrafungsmaßnahmen................................................... 271

Abbildung 5-54: Eignung von emotionalen Konkretisierungsmaßnahmen ................... 274

Abbildung 5-55: Eignung von Individualunterstützungsmaßnahmen.............................. 281

Abbildung 5-56: Eignung von Gruppenunterstützungsmaßnahmen.............................. 286

Abbildung 5-57: Eignung von Beratungsmaßnahmen....................................................... 289

Abbildung 5-58: Personalanzahl-Projektdauer-Verhältnis ................................................. 306

Abbildung 5-59: Motivationsverlauf in Abhängigkeit von der Einstellung...................... 309

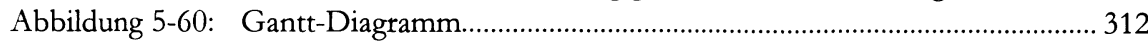

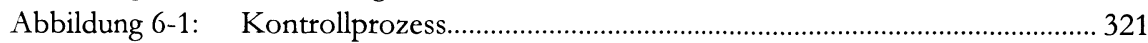




\section{Tabellenverzeichnis}

Tabelle 2-1: Problemursachen gescheiterter Implementierungen von Alexander .............. 47

Tabelle 2-2: Implementierung einer Geschäftsbereichsstrategie ........................................... 57

Tabelle 2-3: Allgemeines Aktionsprogramm auf Funktionsbereichsebene ......................... 59

Tabelle 2-4: Einstellung zu Veränderungen in Abhängigkeit von der Position................... 68

Tabelle 3-1: Strategietypen für den Konsumgütersektor..................................................... 101

Tabelle 3-2: Strategietypen für den Industriegütersektor ..................................................... 102

Tabelle 3-3: Gegenüberstellung von Strategietypen, Zielen und Merkmalen..................... 103

Tabelle 3-4: Gegenüberstellung von Strategiemerkmalen und Strategietypen ................... 104

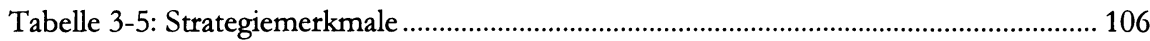

Tabelle 3-6: Kontingenzfaktoren der Differenzierungsstrategie........................................ 109

Tabelle 3-7: Kontingenzfaktoren der Kostenführerschaftsstrategie.................................... 111

Tabelle 3-8: Leistungsdaten der Produktentwicklung........................................................... 114

Tabelle 4-1: Ansoff-Matrix mit Zuordnung von Strategiemerkmalen ................................ 125

Tabelle 5-1: Unterscheidungsmerkmale von Arbeitsgruppen und Teams......................... 217

Tabelle 5-2: Top-10 Lehrmethoden in den USA 1995 ....................................................... 282

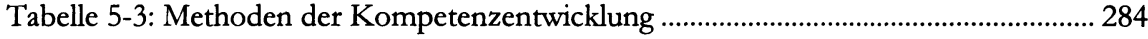

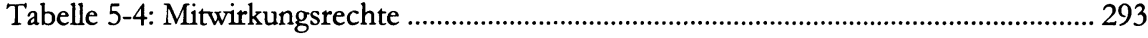

Tabelle 5-5: Rechtliche Restriktionen der Strategieimplementierung................................. 298

Tabelle 5-6: Spezifisches Maßnahmenprogramm von GM/Venezuela .............................. 301

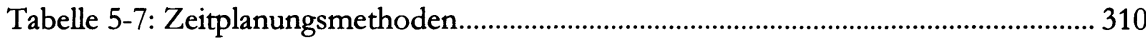

Tabelle 5-8: Work Breakdown Structure .............................................................................. 311

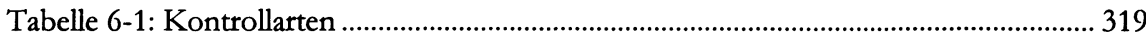




\section{Abkürzungsverzeichnis}

\begin{tabular}{|c|c|}
\hline 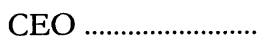 & Chief Executive Officer \\
\hline $\mathrm{CPM}$ & Critical Path Method \\
\hline DBW & Die Betriebswirtschaft \\
\hline 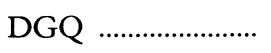 & Deutsche Gesellschaft für Qualität \\
\hline 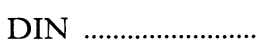 & Deutsches Institut für Normung \\
\hline GE & General Electric \\
\hline 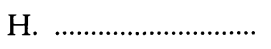 & Heft \\
\hline HWO …...................... & Handwörterbuch der Organisation \\
\hline 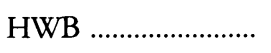 & Handwörterbuch der Betriebswirtschaft \\
\hline MIT …........................ & Massachusetts Institut of Technology \\
\hline PERT .......................... & Program Evaluation and Review Technique \\
\hline ROI …....................... & Return on Investment \\
\hline S. . . & Seite \\
\hline s. a. …….................... & siehe auch \\
\hline SGE & Strategische Geschäftseinheit \\
\hline 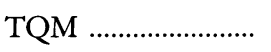 & Total Quality Management \\
\hline WBS …......................... & Work Breakdown Structure \\
\hline WiSt & Wirtschaftswissenschaftliches Studium \\
\hline WISU ……................... & Das Wirtschaftsstudium \\
\hline $\mathrm{ZfB}$ & Zeitschrift für Betriebswirtschaft \\
\hline $\mathrm{ZfbF}$ & $\begin{array}{l}\text { Schmalenbachs Zeitschrift für betriebswirtschaftliche For- } \\
\text { schung }\end{array}$ \\
\hline $\mathrm{ZFO}$ & Zeitschrift Führung und Organisation \\
\hline zit. ............................... & zitiert \\
\hline zugl. ................................ & zugleich \\
\hline
\end{tabular}

